\title{
Cell Division Fidelity Is Altered during the Vascular Response to Injury
}

\section{Its Novel Role in Atherosclerosis Progression}

\author{
Rosalind Silverman-Gavrila, ${ }^{*}$ Lorelei Silverman-Gavrila, ${ }^{\dagger}$ and Michelle P. Bendeck ${ }^{*}$
}

From the Departments of Laboratory Medicine and Pathobiology* and Physiology, ${ }^{\dagger}$ University of Toronto, Toronto, Ontario, Canada

\author{
Accepted for publication \\ November 7, 2012. \\ Address correspondence to \\ Michelle Bendeck, Ph.D., \\ Department of Laboratory \\ Medicine and Pathobiology, \\ Faculty of Medicine, University \\ of Toronto, 1 King's College \\ Cir, Room 6213, Toronto, ON, \\ Canada M5S 1A8. E-mail: \\ michelle.bendeck@utoronto.ca.
}

\begin{abstract}
The rapid proliferation of smooth muscle cells (SMCs) contributes to atherosclerotic plaque formation and neointimal thickening in other occlusive vascular diseases. In cancer cells, rapid cell proliferation is often accompanied by DNA damage, division aberrations, elevated cell apoptosis, or accumulation of abnormal cells. However, little is known about division fidelity in vascular disorders. We have analyzed the cell division fidelity during the rapid SMC proliferation that occurs after balloon injury of the rat carotid artery using en face confocal microscopy of the full thickness of the vessel wall. SMCs newly migrated to the neointima had increased division defects and increased apoptosis compared with SMCS in the subjacent media, despite comparable mitosis rates. Protein kinase $C \alpha$ and the receptor for hyaluronic acid-mediated motility (RHAMM) regulate division fidelity in cultured neointimal SMCs. The centrosomal targeting sequence of RHAMM was required for localization to the mitotic spindle and spindle organization. Dynein and RHAMM colocalized in the spindle area and were part of a complex. Dynein inhibition caused spindle defects similar to RHAMM or protein kinase C inhibition. Our study uncovered abnormalities in rapidly proliferating SMCS after arterial injury that could contribute to the growth of atherosclerotic plaques and reduce plaque stability by triggering apoptosis, and it described a mechanism by which RHAMM and dynein coordinate division fidelity in neointimal SMCs. (Am J Pathol 2013, 182: 628-639; http://dx.doi.org/10.1016/j.ajpath.2012.11.007)
\end{abstract}

The rapid proliferation of smooth muscle cells (SMCs) contributes to the growth of intimal vascular lesions and is inextricably linked to the pathogenesis of atherosclerosis, restenosis, and other occlusive vascular diseases. ${ }^{1}$ Rapid cell proliferation is often accompanied by DNA damage and aberrations in the fidelity of cell division; however, there are few reports on the fidelity of cell division in atherosclerosis. Such disordered cell divisions can lead to an elevated rate of apoptosis in a response to remove the abnormal cells. This phenomenon is important in vascular disease, because the death of SMCs by apoptosis has been linked to plaque instability and rupture, the leading causes of cardiovascular mortality. By contrast, division fidelity is well studied in cancer research, where it is known that defects in division lead to the accumulation of abnormal cells, and the failure to clear these cells via apoptosis contributes to tumorigenesis. Cancer and vascular disease share common causative factors that may affect cell division fidelity, including elevated levels of oxidative stress, alterations in cell adhesion molecules, proteases, and ligand-growth factor receptor interactions; previous investigators have postulated that atherosclerotic plaques are conditional benign neoplasms. ${ }^{2-5}$ More recent studies have drawn attention to the relationship between DNA damage and atherosclerosis, ${ }^{6}$ and we propose that division defects might also be frequent in vascular disease.

Tight control of the number, function, and position of the centrosomes is required for normal cell division and migration. The centrosomes polarize the cytoskeleton in migrating cells

Supported by an operating grant from the Heart and Stroke Foundation of Ontario (T6084 to M.P.B.), a Heart and Stroke Foundation Junior Fellowship (L.S.-G.), and a Canadian Institutes of Health Research grant (82827 to Milton Charlton, University of Toronto, Toronto, ON, Canada). 
and form the poles of the division spindle that drive chromosome segregation. In rapidly proliferating cancer cells, centrosome hyperamplification leads to multipolar spindles, which can lead to chromosome loss or gain at each cell cycle. ${ }^{7,8}$ Previous work from our laboratory has defined a central role for protein kinase $\mathrm{C} \alpha(\mathrm{PKC} \alpha)$ and the receptor for hyaluronic acid-mediated motility (RHAMM) in controlling centrosomal position and functions during migration. We reported that the phosphorylation of RHAMM by PKC $\alpha$ was important to maintain the position of the centrosome behind the nucleus in SMCs migrating into the neointima after arterial injury. ${ }^{9}$ Others have identified critical roles for RHAMMmediated phosphatidylinositol 3-kinase-dependent Rac activation in migration ${ }^{10}$ and for RHAMM in cell division. ${ }^{11}$ We extend our studies to determine whether RHAMM is involved in the control of centrosome position and proper cell division in neointimal SMCs.

By using en face confocal microscopy to image the full thickness of the vessel wall after balloon catheter injury of the rat carotid artery, we assessed the fidelity of cell division and measured apoptosis rates in SMCs. We show an increased frequency of division spindle defects (multipolar spindles and centrosome fragmentation and detachment) and cytokinesis defects in the SMCs from the neointima and in immediately subjacent media. By using in vitro cell cultures, we demonstrated that PKC and RHAMM coordinate events of division, such as spindle organization and stability, balanced karyokinesis, and cytokinesis in neointimal SMCs, with RHAMM acting via its centrosomal targeting sequence and interaction with dynein. To our knowledge, this study is among the first to address the fidelity of cell division in response to vascular injury, and, as such, has potential therapeutic implications for the control of atherogenesis.

\section{Materials and Methods}

\section{Balloon Catheter Injury of the Rat Carotid Artery}

Animal experiments were performed in accordance with the guidelines of the Canadian Council on Animal Care and the University of Toronto Advisory Committee on Animal Care. Six male Sprague-Dawley rats (Charles River, Montreal, PQ, Canada) were anesthetized by i.p. injection, and common carotid arteries were injured by passing a $2 \mathrm{~F}$ embolectomy balloon catheter along their length to denude the endothelium, three times, as previously described. ${ }^{12}$ Four days later, the carotids were perfusion fixed with $4 \%$ paraformaldehyde for 15 minutes, excised, cut longitudinally, and pinned with the intimal surface up. Six arteries were permeabilized with $0.2 \%$ Triton-X100 for 5 minutes, treated with $100 \mu \mathrm{g} / \mathrm{mL}$ RNAase A for 1 hour, and incubated for 1 hour with a 1:200 dilution of a mouse monoclonal anti- $\gamma$-tubulin antibody clone, GTU-88 (Sigma-Aldrich, St. Louis, MO), with a 1:50 dilution of Alexa 488 goat anti-mouse antibody (Molecular Probes, Life Technologies, Carlsbad, CA), and for 20 minutes with $20 \mu \mathrm{g} / \mathrm{mL}$ propidium iodide (PI; Molecular Probes, Life
Technologies). To confirm that the analyzed cells were SMCs, we stained artery segments for smooth muscle $\alpha$-actin with a mouse monoclonal antibody, A2547 (Sigma-Aldrich), and for Smemb (nonmuscle major histocompatibility complex) (Abcam, Cambridge, MA), and counterstained with Hoechst and PI, respectively, for DNA identification.

\section{Cell Culture and Inhibitor Treatments}

To obtain medial SMCs, uninjured carotid arteries were stripped of adventitia and the endothelium was scraped off; then, medial SMCs were dispersed by digestion, as previously described. ${ }^{13}$ To obtain neointimal SMCs, left rat carotid arteries were injured with a balloon catheter, and 2 weeks later, the thickened neointima was stripped from the vessel, and cells were dispersed. ${ }^{13}$ Cells were grown to confluence, then subject to a scrape wound, as previously described. ${ }^{9}$ Some cells were treated with $1 \mu \mathrm{mol} / \mathrm{L}$ PKC inhibitor, bisindolylmaleimide I (Calbiochem, San Diego, $\mathrm{CA})$, at 30 minutes before wounding and continued for 6 hours after wounding. Some cells were treated with 25 $\mu \mathrm{mol} / \mathrm{L} \mathrm{Na}_{3} \mathrm{VO}_{4}$ (vanadate) for 30 minutes before wounding and continued for 6 hours after wounding.

\section{TUNEL Assay}

Paraffin-embedded sections from rat carotid arteries at 6 hours and 4, 7, 10, and 14 days after injury or uninjured controls were labeled with the ApopTag Plus kit (EMD; Millipore, Billerica, MA).

\section{siRNA-Targeting RHAMM}

Neointimal SMCs were transfected with validated siRNAsequences (Ambion Biosciences, Austin, TX): RHAMMspecific (Silencer Predesigned siRNA ID No. 48767) and nontargeting siRNA Silencer Negative control. ${ }^{9}$ After siRNA treatment, some cells were transfected with mutant or wild-type (WT) RHAMM-green fluorescent protein (GFP) plasmid. A total of $25 \times 10^{5}$ cells that were $50 \%$ to $80 \%$ confluent were plated on coverslips in 6-well plates in 2 $\mathrm{mL}$ of Dulbecco's modified Eagle's medium with $10 \%$ fetal calf serum with no antibiotics. For each well, $2.5 \mu \mathrm{g}$ of plasmid DNA was dissolved in $500 \mu \mathrm{L}$ of Opti-MEM I reduced serum medium. After 5 minutes, $15 \mu \mathrm{L}$ per well of transfection agent (DharmaFect Duo; ThermoFisher, Nepean, ON, Canada) was added. Thirty minutes later, 500 $\mu \mathrm{L}$ of DNA-transfection agent complexes was added; 24 hours later, the cells were tested for the appearance of the GFP signal. Cells were wounded at confluence with a needle to generate an 80- to 100- $\mu \mathrm{m}$ wide wound and were fixed 6 hours later. WT or mutated RHAMM sequences that had the amino acids in the centrosome-targeting domains replaced by alanine residues were cloned into the $\mathrm{Xhol} / \mathrm{HindIII}$ sites of the pEGFP-N1 vector (Clontech, Mountain View, CA) by Genscript (Piscataway, NJ). 


\section{Western Blot Analysis}

Cell lysates were probed with rabbit polyclonal anti-RHAMM antibody H-90 (Santa Cruz Biotechnology Inc., Santa Cruz, CA), diluted 1:1000, and goat anti-rabbit IgG horseradish peroxidase-conjugated antibody (Upstate Biotechnology; EMD Millipore, Billerica, MA). The membranes were stripped with stripping buffer for 30 minutes $[0.1 \mathrm{~mol} / \mathrm{L}$ glycine- $\mathrm{HCl}(\mathrm{pH} 2.5$ to 3.0)] and reprobed with mouse monoclonal anti- $\alpha$-tubulin antibody, diluted 1:1000, as a control for loading variations.

\section{Co-Immunoprecipitation}

Immunoprecipitations were performed using $10 \mathrm{mg} / \mathrm{mL}$ of mouse monoclonal anti-intermediate chain dynein antibody (Millipore, Billerica, MA) or rabbit polyclonal antibody for RHAMM (Santa Cruz Biotechnology Inc.) and protein G agarose beads.

\section{Fluorescence Staining}

All cultured SMCs were fixed for 2 minutes in methanol, with the exception of those stained for F-actin that were fixed 20 minutes with $4 \%$ paraformaldehyde. ${ }^{9}$ Cells were incubated for 1 hour with primary antibodies [goat anti-rabbit polyclonal antibody for $\gamma$-tubulin, diluted 1:100 (SigmaAldrich), goat anti-mouse monoclonal antibody for $\alpha$-tubulin clone DM1-A, diluted 1:700 (Sigma-Aldrich), mouse monoclonal antibody for $\gamma$-tubulin clone GTU-88, diluted 1:200 (Sigma-Aldrich), rabbit polyclonal antibody for RHAMM (Santa Cruz Biotechnology Inc.), diluted 1:100, or mouse monoclonal anti-intermediate chain dynein antibody, diluted 1:100] and corresponding secondary antibodies, diluted 1:50 [Alexa 568 or 488 goat anti-rabbit or Alexa 568 or 488 goat anti-mouse, respectively (Life Technologies, Carlsbad)]. Some cells were incubated with $2 \mu \mathrm{mol} / \mathrm{L}$ Alexa 568-labeled phalloidin (Sigma-Aldrich), and all cells were counterstained for DNA with Hoechst, $2 \mu \mathrm{g} / \mathrm{mL}$ (Molecular Probes, Life Technologies) for 10 minutes.

Confocal microscopy was performed with an Olympus Fluoview 1000 confocal microscope (Olympus, Richmond Hill, ON, Canada) or with a Nikon Eclipse Ti-E (Nikon, Mississauga, ON, Canada) and a $60 \times$ oil lens (numerical aperture, 1.4). Images represent collapsed 15 to $20 \mathrm{Z}$-series of $0.2-\mu \mathrm{m}$ steps.

The cell proliferation rate was assessed based on quantification of the mitotic index (ie, number of cells in mitosis/ total cell count). DNA staining was used for identification of cells in mitosis and interphase.

\section{Statistical Analysis}

Means and SDs were calculated with Excel (Microsoft, Redmond, WA), and the Student's $t$-test was performed with Sigma Stat, version 3.1 (Systat Software Inc., Point Richmond, CA).

\section{Results}

Mitotic Index and Division Defects Are Higher in SMCs from the Neointima and Immediately Subjacent Medial Areas of Rat Carotid Arteries

We studied arteries harvested 4 days after balloon catheter injury of the rat carotid artery, examining SMCs in the neointima and media using en face preparations imaged by confocal microscopy. We measured SMC mitoses in the following areas: i) SMCs newly arrived in the neointima; ii) the medial layer underneath; iii) medial layers distant from the sites of neointimal SMCs, where neointimal SMC migration had not yet occurred; and iv) the media of uninjured arteries. A three-dimensional reconstruction of the carotid artery imaged by confocal microscopy showed SMC nuclei in the neointima and underlying medial SMC nuclei (Figure 1A). Natural green autofluorescence of elastin in the internal elastic laminae marked the boundary between the medial and intimal layers. Five to seven Z-steps of confocal images were collapsed to discriminate these layers independently. Mitotic figures were present in the neointima (Figure 1B) and the medial layers just beneath the neointima (Figure 1C), with a higher frequency than in the medial area of injured arteries where no migration of SMCs had yet occurred, or in the medial areas in control uninjured vessels (Figure 1D). The most frequent division defects were micronuclei (Figure 1, E and F), multicentrosomal mitotic figures (Figure 1, G and $\mathrm{H}$ ), and binucleate cells (Figure 1, I and $\mathbf{J}$ ). The cell division defect frequency was higher in the neointima and in the media just beneath the neointima than in the other layers of arteries, and defects occurred at a higher rate in the neointima than in the subjacent medial SMCs (Figure 1K). To confirm that the cells analyzed for division defects were SMCs, we stained artery segments with a monoclonal antibody against smooth muscle $\alpha$-actin (Figure 1, L and M), and for Smemb (nonmuscle myosin heavy chain) (Figure 1, N and O). Segments were counterstained for DNA to visualize cell division defects. Both SMC markers stained cells with defects, such as binucleate cells (Figure 1, L-O), cells with micronuclei (Figure 1, $\mathrm{L}-\mathrm{O}$ ), or multiple centrosome mitotic figures (Figure 1, $\mathrm{N}$ and $\mathrm{O}$ ). These results suggested that the cells analyzed were SMCs.

\section{SMC Apoptosis Increases to 14 Days after Injury}

Cross sections of balloon-denuded carotid arteries, taken at various times after injury, were stained with TUNEL to mark apoptotic cells and with PI to label nuclei. TUNELpositive cells were not present in control uninjured arteries (Figure 2A), and were first observed at 7 days in the neointimal and medial layers (Figure 2, B and $\mathrm{C}$ ). 

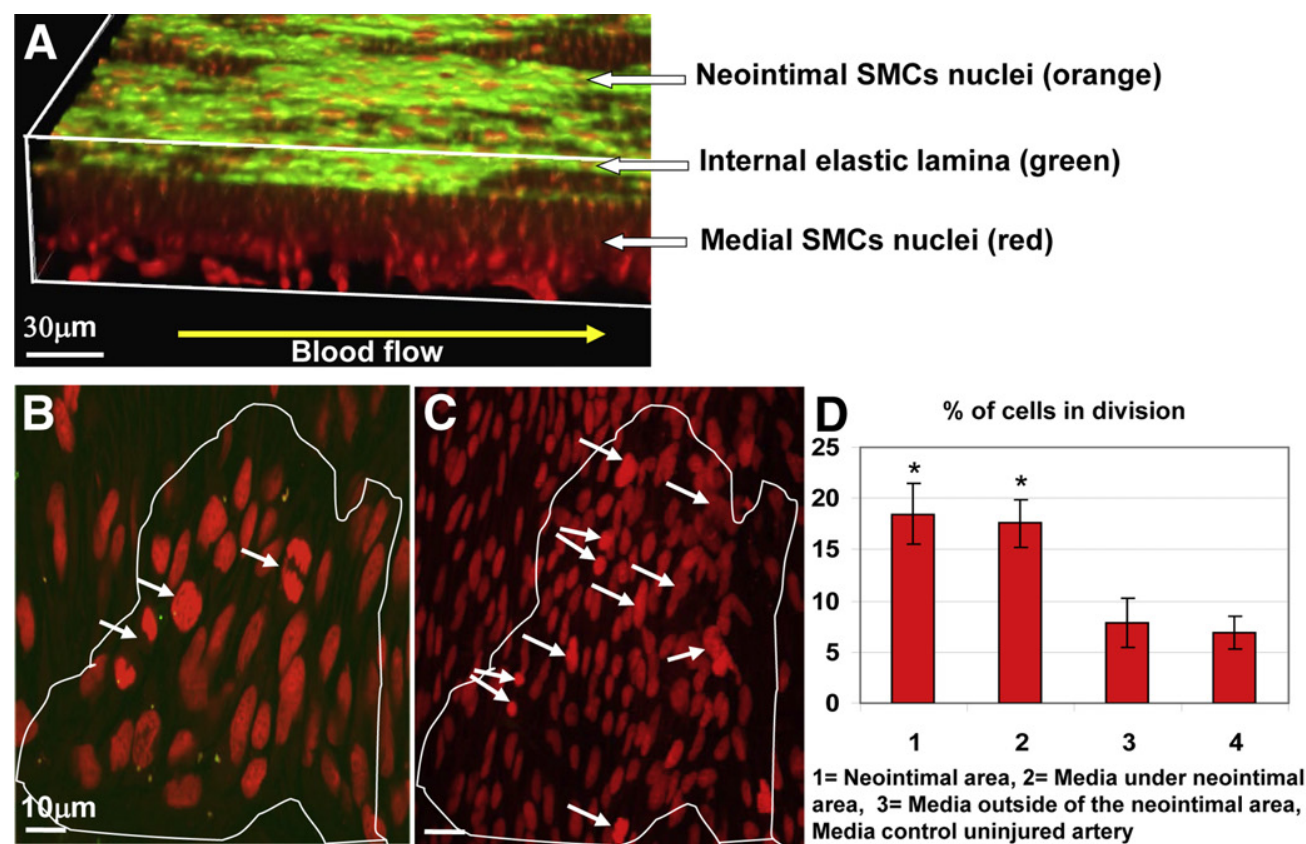

$1=$ Neointimal area, $2=$ Media under neointimal area, $3=$ Media outside of the neointimal area, $4=$ Media control uninjured artery
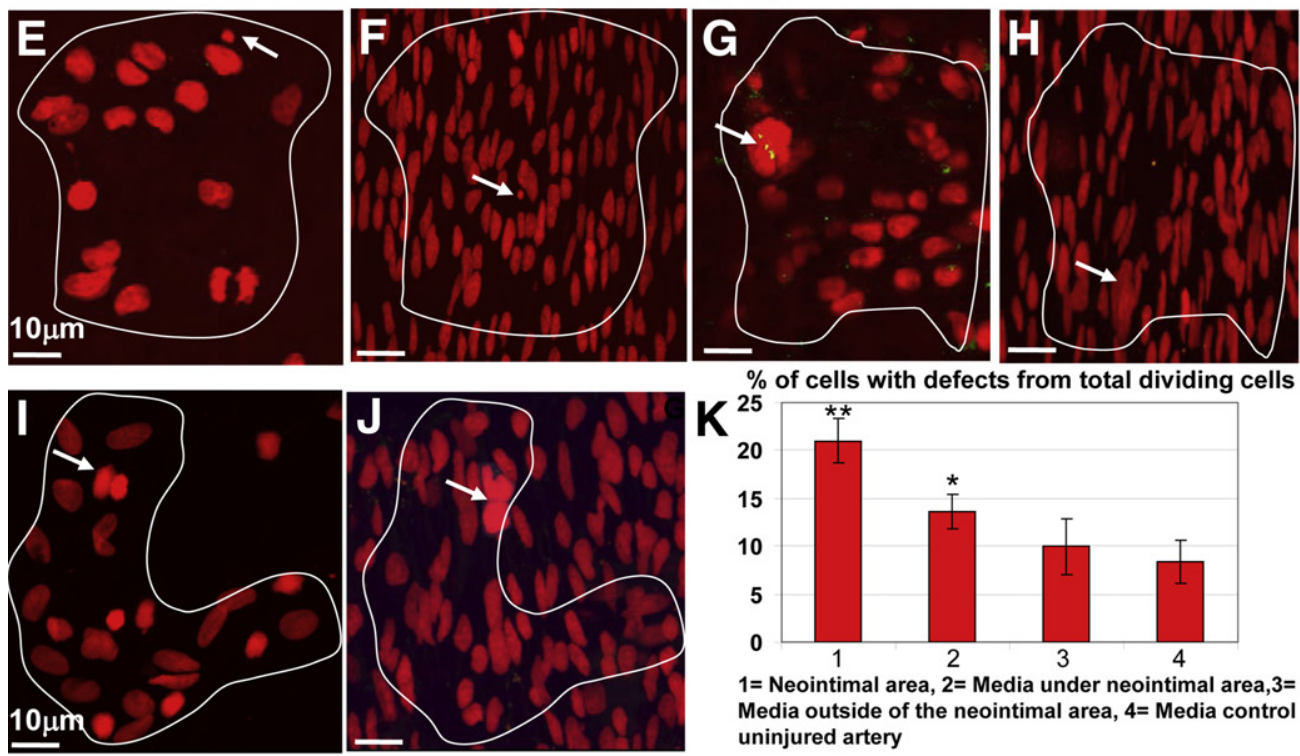

$1=$ Neointimal area, $2=$ Media under neointimal area, $3=$ Media outside of the neointimal area, $4=$ Media control uninjured artery
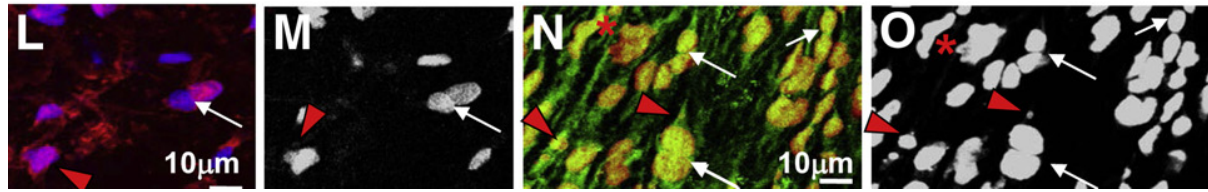

Figure 1 The proliferation rate and cell division defect frequency at 4 days after balloon injury are higher in the neointima and immediately subjacent medial layers. A: Three-dimensional reconstruction of a projection of 10 Z-steps of a control artery imaged en face showing nuclei of SMCs (orange-red) that have migrated through the elastic lamina. The internal elastic lamina is seen (green) because of autofluorescence. B, C, E-J: Confocal microscopy images of rat arteries stained for DNA (red) and for centrosomes (green). White lines delimitate an area where cells were counted in media and neointima. Arrows indicate nuclei in mitosis. B: SMCs in the neointima. C: SMCs in the medial layer beneath the neointima. D: The mitotic index is higher in the neointima and the medial layer just beneath the newly formed neointima than in the rest of media and in the media of uninjured arteries. ${ }^{*} P \leq 0.05$ comparing 1 or 2 with 3 or 4 . E: SMCs in the neointima with micronucleus (arrow). F: SMCs in the medial region under the neointima with micronucleus (arrow). G: SMCs in the neointima with a multicentrosomal mitotic figure (arrow). H: SMCs in the medial region beneath the neointima, with a multicentrosomal mitotic figure (arrow). I: SMCs in the neointima with a binucleate cell (arrow). J: SMCs in the medial region beneath the neointima with a binucleate cell (arrow). K: The frequency of division defects is higher in the neointima than in the media just beneath the neointimal area, than in the rest of the medial layer, and in the media of uninjured arteries. $N=20$ repeats of four-area measurements, and $n=$ counted SMCs: 786 in the neointima, 932 in the media beneath the neointima, 845 in a medial region outside of the neointimal area, and 727 in uninjured artery areas. ${ }^{*} P \leq 0.05$ comparing 2 with 3 or $4 ;{ }^{*} P \leq 0.05$ comparing 1 with 2 . Data indicate means \pm SD. L: Confocal microscopy images of neointimal SMCs stained for smooth muscle $\alpha$ actin (red) and DNA (blue) with binucleate cells (white arrow) and micronucleus (red arrowhead). M: The DNA channel of L. N: Confocal microscopy images of neointimal SMCs stained for SMemb (green) and DNA (red) with binucleate cells (white arrows), micronuclei (red arrowheads), and a multicentrosomal mitotic figure (red asterisk). 0: The DNA channel of $\mathbf{N}$. 
Apoptosis peaked at 14 days after injury, with $31 \%$ of the cells in the neointima and $18 \%$ of cells in the media staining TUNEL positive (Figure 2, D-F). The apoptotic cells in the medial layer were randomly distributed throughout the wall.

\section{PKC $\alpha$ and RHAMM Are Required for Karyokinesis and Cytokinesis Fidelity in Neointimal, But Not Medial, SMCs}

To elucidate the mechanisms controlling karyokinesis and cytokinesis in SMCs, we used a model of scrape wounding confluent monolayers of neointimal SMCs isolated from injured carotid arteries, and medial SMCs from uninjured carotid arteries. The scrape wounding model mimicks critical features of the response to injury in arteries, including cell proliferation and migration, and is an accepted method to investigate cell division defects and migration in vitro.

Neointimal SMCs followed a typical mitotic cycle (Figure 3, A-E); however, $>65 \%$ of neointimal SMCs exhibited premature division of the centrosome, which occurred during late telophase-cytokinesis (Figure 3, D and E). Normally, in most non-transformed cells, the centrosome divided much later, during the $\mathrm{S}$ phase (interphase) of the next cell cycle. One notable exception is the syncytial, rapid division of Drosophila embryos, in which the centrosomes divide in early anaphase. ${ }^{14}$ This suggests that neointimal SMCs might have an accelerated cell cycle compared with most mitotic cells, which might enable a more rapid proliferation rate. By contrast, medial SMCs did not exhibit premature centrosomal division (Figure 3F).

Our previous research using inhibitors of various PKC isoforms and siRNA for RHAMM established critical roles for $\mathrm{PKC} \alpha$ and RHAMM in modulating the position of the centrosome and directed migration in neointimal SMCs. ${ }^{9}$ To determine whether PKC $\alpha$ regulates centrosomaldependent processes in division, neointimal SMCs were wounded and treated with the selective PKC $\alpha$ inhibitor, bisindolylmaleimide I, for 6 hours, and dividing cells at the wound edge were examined. The percentage of dividing cells with multipolar spindles (tripolar to heptapolar spindles) (Figure 3G), fragmented centrosomes (Figure 3H), and detached centrosomes (Figure 3I) was increased in cells treated with the PKC inhibitor (Figure 3J). Cells with premature replication of the centrosomes in anaphase (Figure $3 \mathrm{~K}$ )
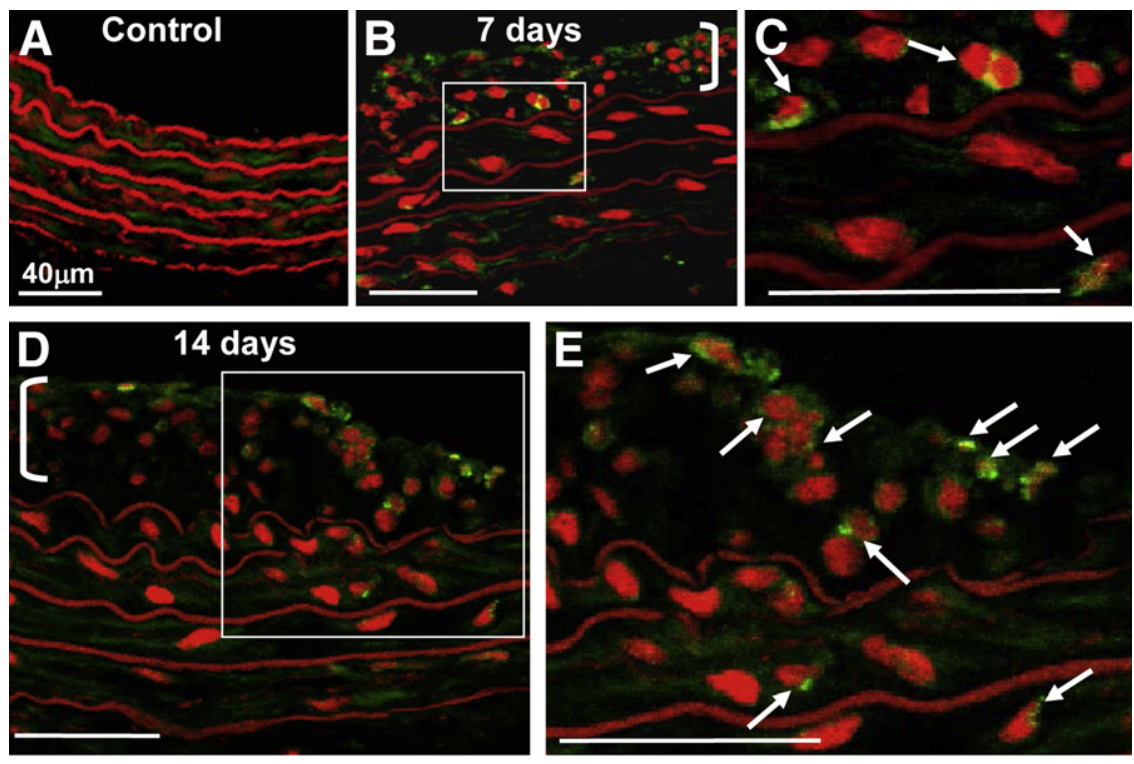

$\mathbf{F}$
$\%$ of TUNEL positive or dividing nuclei

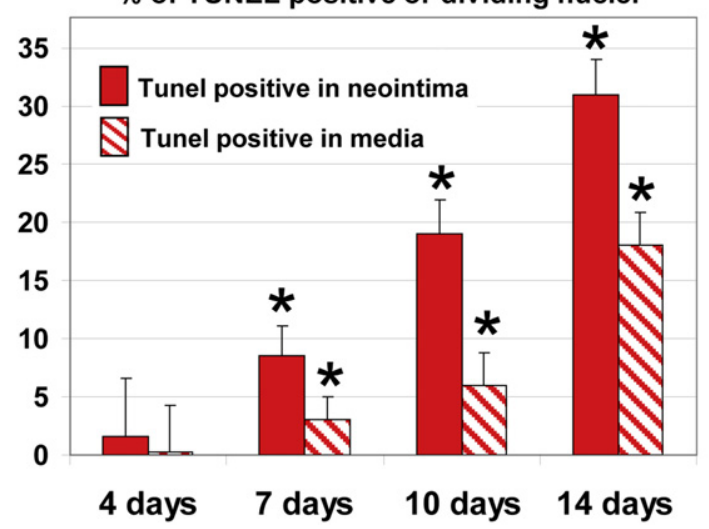

Figure 2 Apoptosis rate increases to 14 days after balloon injury. Confocal microscopy images of cross sections of rat carotid arteries TUNEL stained for apoptotic nuclei (bright green; arrows in C and $\mathbf{E}$ ) and counterstained with PI (red) for nuclei. Collagen autofluorescence is pseudocolored dark green, and elastin autofluorescence is pseudocolored red. The white brackets indicate the thickness of the neointima. A: There was no TUNEL-positive staining in control uninjured arteries. B: TUNELpositive staining was evident mainly in the neointima at 7 days after injury. C: An enlargement of the area within the boxed area in B. D: TUNELpositive staining was evident in the neointima and in the media at 14 days after injury. E: An enlargement of the area within the boxed area in $\mathbf{D}$. F: The percentage of apoptotic cells at various time points after injury. ${ }^{\star} P \leq 0.05$ comparing different time points or between the neointima and media at each time point. Four areas of an artery for each time point and four arteries per time point were analyzed to give a total of $n=980$ cells for control, $n=840$ cells for 6 hours, $n=934$ cells for 4 days, $n=1011$ cells for 7 days, $n=765$ cells for 10 days, and $n=845$ cells for 14 days. Scale bar $=40 \mu \mathrm{m}$. Data in $\mathbf{F}$ indicate means $\pm \mathrm{SD}$. 

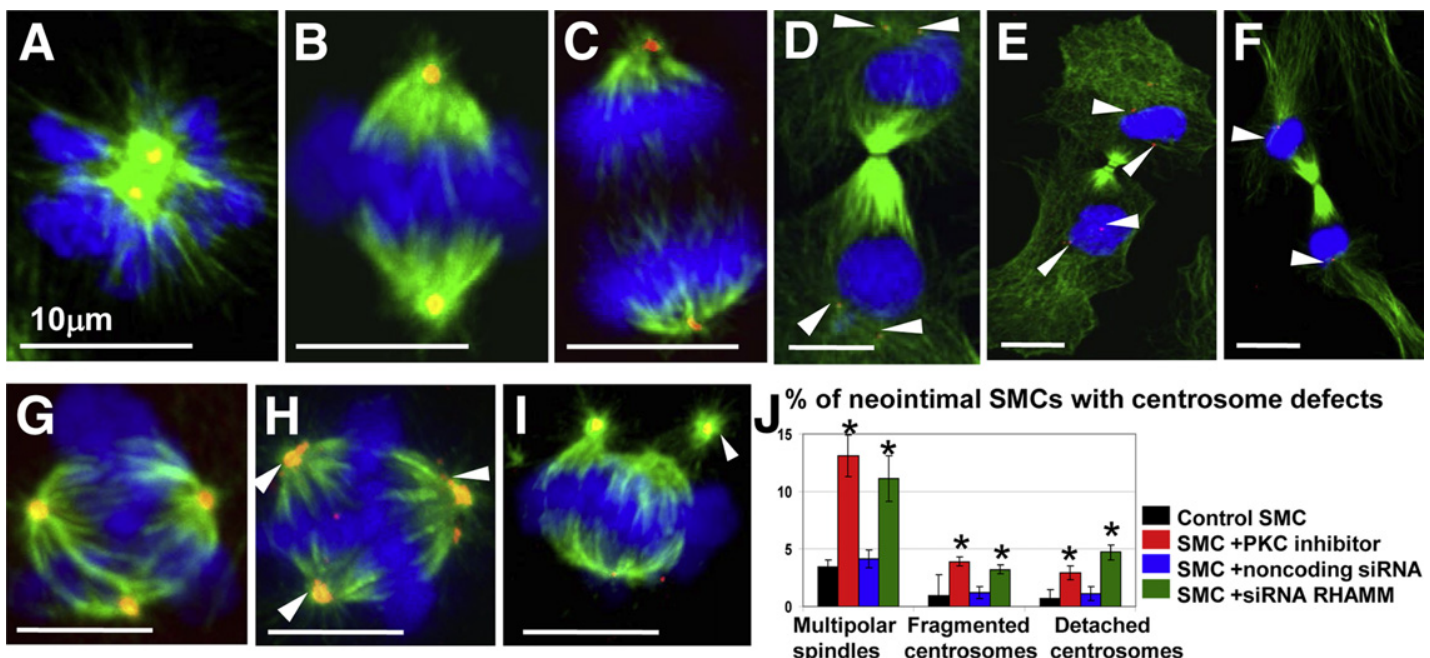

\% of neointimal SMCs with centrosome defects
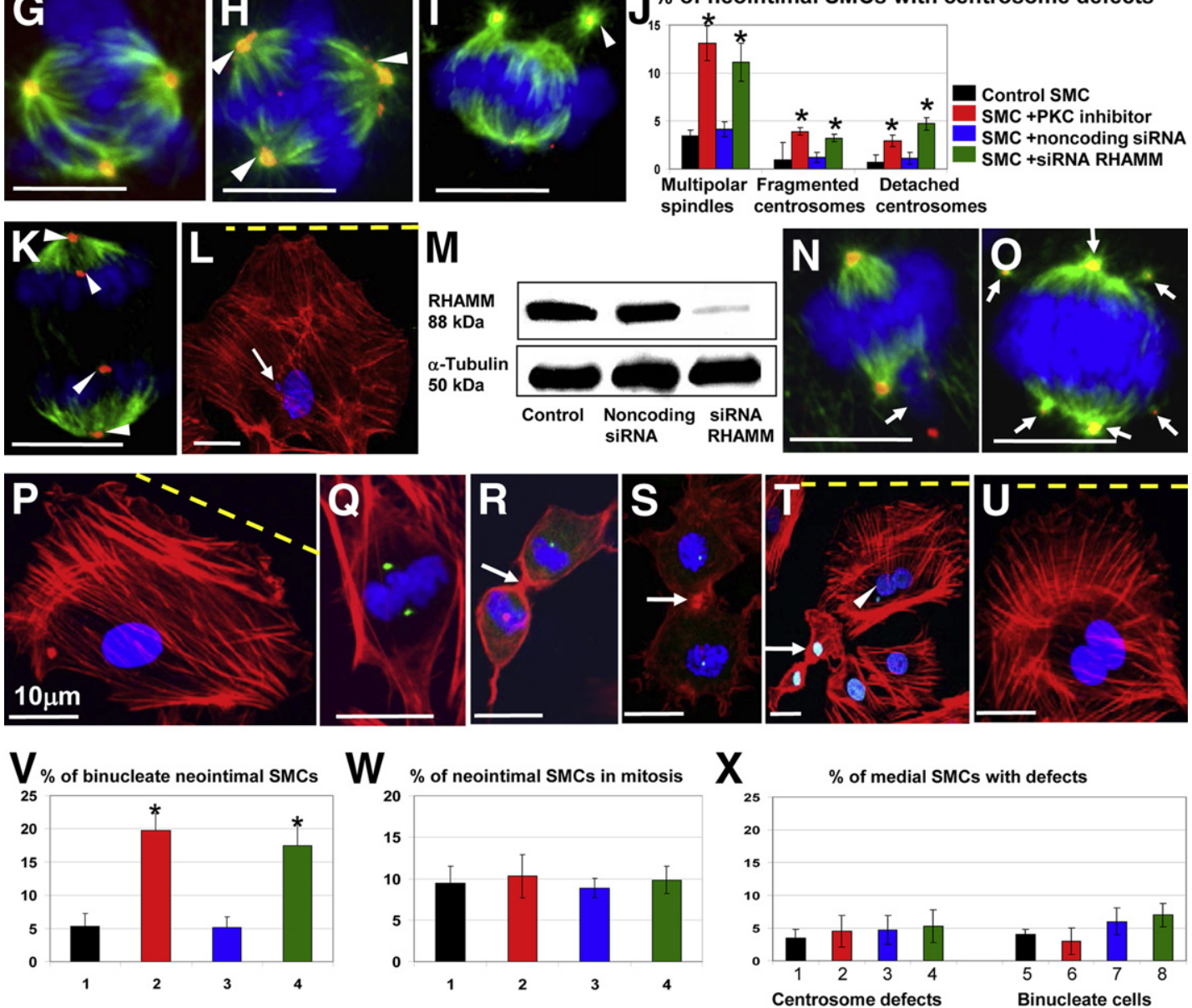

Figure 3 PKC inhibition and siRNA for RHAMM disrupts spindle organization and function and increases the frequency of binucleate cells in neointimal SMCs. A-I, K, N, and 0: Confocal microscopy images of carotid artery SMCs subject to scrape wound injury in vitro: DNA (blue), microtubules (green), and centrosome (red). Yellow or orange indicates colocalization of centrosome and microtubules. The normal phases of mitosis in control neointimal SMCs: prophase (A), metaphase (B), anaphase (C), telophase (premature duplication of centrosomes; arrowheads) (D), and late cytokinesis (premature duplication of centrosomes; arrowheads) (E). F: Cytokinesis in medial SMCs. Treatment with the PKC inhibitor increased the frequency of multipolar spindles $(\mathbf{G})$, fragmented centrosomes $(\mathbf{H})$, and detached centrosomes (I). J: Graph showing the frequency of spindle defects measured after PKC inhibition or RHAMM siRNA. K: Premature duplication of the centrosome in anaphase was frequently observed after PKC inhibition. L and P-U: DNA (blue), centrosome (green), and actin (red). L: Cell with a micronucleus (arrow) after PKC inhibition. M: RHAMM-targeting siRNA reduced expression, as assessed by using Western blot analysis, whereas noncoding siRNA had no effect. N: Lagging chromosome (arrow) does not attach to a multicentrosomal spindle with pseudobipolar architecture after treatment with RHAMM siRNA. 0: Clustering of multiple centrosomes (arrows) generates a broad bipolar spindle after siRNA for RHAMM. P-S: Control cells stained to show the actin cytoskeleton and centrosome during the cell cycle. P: Interphase. Q: Metaphase. R: Cytokinesis. Arrow indicates actin in the contractile furrow. S: Abscission. Arrow indicates the place of cell severing. T: Control neointimal SMCs contain a few binucleate cells (arrowhead). Arrow indicates a cell in cytokinesis. U: Binucleate cell after PKC inhibition. V: The frequency of binucleate cells is higher after PKC inhibition or siRNA for RHAMM. W: Neither PKC $\alpha$ inhibition nor siRNA for RHAMM affected cell mitotic indexes. X: Neither PKC $\alpha$ inhibition nor siRNA for RHAMM caused a significant increase in centrosomal defects or binucleate cells in medial SMCs. Neointimal SMCs stained for $\alpha$-tubulin and $\gamma$-tubulin: $n=665$ control, $n=757$ PKC inhibitor treated, $n=506$ noncoding siRNA treated, and $n=712$ siRNA RHAMM treated. Neointimal SMCs stained for actin and $\gamma$-tubulin: $n=278$ control, $n=327$ PKC inhibitor treated, $n=366$ noncoding siRNA treated, and $n=474$ siRNA RHAMM treated. Medial SMCs stained for $\alpha$-tubulin and $\gamma$-tubulin: $n=412$ control, $n=521$ PKC inhibitor treated, $n=308$ noncoding siRNA treated, and $n=498$ siRNA RHAMM treated. $N=6$ repeats of the experiment. The yellow dashed line $(\mathbf{L}, \mathbf{P}, \mathbf{T}$, and $\mathbf{U})$ indicates the wound. Scale bar $=10 \mu \mathrm{m}$. ${ }^{*} P<0.05$. Data indicate means \pm SD. 
and formation of micronuclei (Figure 3L) were also observed after PKC $\alpha$ inhibition.

To study the functions of RHAMM in cell division and karyokinesis, cells were transfected with targeting siRNA, and compared with cells transfected with noncoding siRNA. The knockdown of RHAMM was confirmed by using Western blot analysis (Figure 3M). After RHAMM knockdown, the frequency of multipolar spindles and fragmented and detached centrosomes increased compared with cells transfected with noncoding siRNA (Figure 3J). Premature centrosome replication and micronuclei were also noted after RHAMM knockdown, similar to after PKC inhibition (data not shown). We also noticed some cases of anaphase with lagging chromosomes in multicentrosomal spindles with a pseudobipolar architecture (Figure $3 \mathrm{~N}$ ) after inhibition of PKC (in $1.2 \% \pm 1 \%$ of cells) or RHAMM (in $1.7 \% \pm$ $0.8 \%$ ). We also observed clustering of multiple centrosomes in a rough line with formation of broader spindles (Figure 3O) after inhibition of PKC (in 1\% $\pm 1 \%$ of cells) or RHAMM (in $0.9 \% \pm 0.9 \%$ ).

We next assessed whether RHAMM and PKC $\alpha$ were required to maintain the fidelity of cytokinesis in neointimal SMCs. During division, actin filaments reorganize from an interphase distribution (Figure 3P), to a cortical distribution around the dividing chromosomes (Figure $3 \mathrm{Q}$ ), and into the contractile furrow at the cell equator in late anaphase (Figure 3R). The furrow pinches the cell, dividing it into two equal mononucleate daughter cells after abscission (Figure 3S). Binucleate cells are the result of failed cytokinesis (Figure 3, T and $\mathrm{U}$ ). The baseline frequency of binucleate daughter cells was approximately 5\% in neointimal SMCs, and this increased significantly after PKC inhibition or RHAMM knockdown (Figure 3V).

We determined whether RHAMM or PKC $\alpha$ inhibition affected mitotic indexes in cultured neointimal SMCs. In control SMCs, $9.4 \% \pm 2 \%$ of the cells were in mitosis, whereas in cells treated with noncoding siRNA, $8.86 \% \pm$ $1.18 \%$ of the cells were in mitosis. Neither treatment with PKC inhibitor $(P=0.613)$ nor with siRNA for RHAMM $(P=0.233)$ caused a significant change in the mitotic index (Figure $3 \mathrm{~W}$ ). The data described suggested that RHAMM and $\mathrm{PKC} \alpha$ regulate division fidelity but do not affect cell entry into mitosis.

We also tested the PKC $\alpha$ inhibitor and siRNA for RHAMM on medial SMCs harvested from uninjured carotid arteries, but neither treatment affected the percentages of cells with centrosomal defects $(P=0.686$ and $P=0.775$, respectively) nor those that were binucleated $(P=0.251$ and $P=0.637$, respectively) (Figure $3 \mathrm{X}$ ). The fact that medial SMC division was not affected by PKC $\alpha$ or RHAMM inhibition suggests that this pathway to control division fidelity is unique to neointimal SMCs, and ascertains that this is not merely a general phenomenon displayed by cells in tissue culture. In sum, RHAMM and PKC $\alpha$ control bipolar spindle assembly and spindle pole organization, which ensures the fidelity of chromosome segregation and cytokinesis in neointimal SMCs.

\section{The Centrosomal-Targeting Domain of RHAMM Is Required for Neointimal SMC Division Fidelity}

Next, we performed experiments to study the localization of RHAMM in dividing and migrating SMCs. Staining for RHAMM and $\alpha$-tubulin (Figure 4A) or $\gamma$-tubulin (Figure 4B) demonstrated that, during mitosis, RHAMM colocalized with spindle microtubules, especially near the spindle poles. In interphase migrating cells, RHAMM was in the cytoplasm, concentrated in the perinuclear area (Figure 4, C and D).

The centrosomal-targeting domain of human RHAMM is at the carboxyterminal end, ${ }^{11}$ and overlapped two hyaluronan-binding domains located at amino acids 401 to 411 and 423 to $432 .{ }^{15}$ The human and rat RHAMM sequences in this domain differ by only two amino acids, at 426 and 431 . We generated a GFP-tagged RHAMM mutant by replacing all amino acids in the centrosome-targeting domain with alanine, and investigated whether this mutation altered its localization and function in the division spindle pole. We first treated neointimal SMCs with siRNA to knock down RHAMM, and then transfected cells with either the WT RHAMM-GFP construct to rescue the cells or the RHAMMGFP mutant. During interphase, WT RHAMM-GFP localized in the cytoplasm, with enrichment in the perinuclear area (Figure 4E). By contrast, mutant RHAMM-GFP levels were reduced in the cytoplasm and were not concentrated in the perinuclear area (Figure 4F). Spindle pole defects were infrequent in normal cells and in cells treated with siRNA for RHAMM and transfected with WT RHAMM-GFP (Figure 4G), and RHAMM was localized normally in these cells (Figure $4 \mathrm{H}$ ). By contrast, in cells treated with the mutant RHAMM-GFP, the frequency of spindle pole defects was increased (Figure 4G); in association with this, there was less RHAMM localized around centrosomes (Figure 4I).

RHAMM and Dynein Colocalized in the Spindle and Reciprocal Co-Immunoprecipitation Demonstrate that RHAMM Interacts with Dynein in a Complex

Previous studies have showed that RHAMM interacts with dynein and plays an important role mediating spindle organization in Xenopus and HeLa extracts. ${ }^{11}$ Accordingly, we investigated interactions between RHAMM and dynein in vascular SMCs. Immunofluorescence staining showed that RHAMM and dynein colocalized in the spindles of neointimal SMCs at the spindle poles and on kinetochore microtubules (Figure 4J), suggesting that the two proteins interacted. To further verify whether the two proteins are part of a complex, reciprocal co-immunoprecipitation studies were performed with cell lysates. After lysis, proteins were immunoprecipitated with either anti-dynein or RHAMM antibody and then immunoblotted using an anti-RHAMM or 
an anti-dynein antibody, respectively. RHAMM (88 kDa) was pulled down in immunoprecipitates for dynein, and conversely dynein $(70 \mathrm{kDa})$ was pulled down in immunoprecipitates for RHAMM (Figure 4K).

\section{Dynein Is Required for Spindle Organization and Chromosome Segregation}

To investigate the role that dynein plays in spindle assembly and division, cells were treated with $25 \mu \mathrm{mol} / \mathrm{L} \mathrm{Na}_{3} \mathrm{VO}_{4}$ (vanadate) for 6 hours after wounding. This concentration has been shown to inhibit cytoplasmic dynein motor activity. ${ }^{16}$ Dynein inhibition in neointimal SMCs led to the development of multipolar spindles (Figure 4, L, M, N, and P), broader spindles (Figure 4L), detached centrosomes (Figure 4, L, M, and $\mathrm{P}$ ), and lagging chromosomes in multicentrosomal spindles with a pseudobipolar architecture (Figure 4, N and P). These defects were similar to defects observed after treatment of SMCs with the PKC $\alpha$ inhibitor or siRNA to knock down RHAMM (Figure 3). However, there were some differences. After dynein inhibition, there were more bipolar spindles with lagging chromosomes (Figure 4, O and P), a defect not seen in controls or cells treated with PKC $\alpha$ inhibitor or siRNA for RHAMM. Centrosome fragmentation was less frequent after dynein inhibition (Figure 4P) compared with cells treated with PKC inhibitor or siRNA for RHAMM $(P=0.002)$ (Figure 3J). Taken together, our data suggests that RHAMM, PKC, and dynein act together to regulate bipolar spindle assembly, focusing microtubules at spindle poles and centrosome connection to division spindles.

\section{Discussion}

As expected, we found that the mitotic index was higher in cells that migrated through the elastic lamina to form the neointima and in the adjacent medial layer just beneath it, compared with SMCs in other areas of the media, or in uninjured arteries. We also observed defects in spindle assembly, chromosome division, and cytokinesis in the neointima. It is common for the frequency of division defects to increase with the rate of mitosis. However, neointimal SMCs and subjacent medial SMCs had comparable mitosis rates, yet there were more division defects in the neointimal SMCs, suggesting that there may be unique factors acting on these cells once they migrate through the elastic lamina to hinder proper division. We speculate that the mechanical stress of passing through fenestrae in the internal elastic lamina and/or the exposure to the shear stress exerted by blood flow may trigger mechanotransduction signaling that leads to alterations in cytoskeletal components and, ultimately, to defects in spindle organization and cell division defects. Cell rounding at the onset of mitosis, which depends on moesin activation and the establishment of a rigid actin cortex, is a key element for proper spindle assembly and well-orchestrated chromosome segregation. ${ }^{17}$ Mechanical stress may be transduced by the rigid actin cortex, or it may alter cortex rigidity and thereby influence division fidelity.

Defects in DNA segregation are highly correlated with programmed cell death. Because division defects accumulating in the neointima could trigger the apoptotic machinery, we measured the rate of apoptosis in the neointima and media, and showed that the frequency of apoptosis was much higher in the neointima than in the media, and was maximal at 14 days after balloon injury. The SMCs within the luminal-most layers of the intima continue to proliferate long after injury, ${ }^{18}$ and we observed that these cells also accumulate spindle defects and have increased apoptosis. This is in accord with previous studies that have shown apoptosis after balloon injury in this model. ${ }^{19,20}$ This could lead to ongoing erosion of cells from the surface of the neointima. We propose that a similar situation may occur in human atherosclerosis, in which SMCs exposed to the flowing blood die and are eroded from the surface, leading to plaque instability and rupture. One limitation of the current study is that we used a model of complete endothelial denudation, which probably never occurs in human atherosclerosis. However, there is evidence for denudation of focal areas of the endothelial cell monolayer in atherosclerosis and disruption of large areas of endothelium after angioplasty and stenting. In either case, it is likely that SMCs migrating into the intimal layer are exposed directly to high levels of shear stress; thus, our model and results are relevant.

Our data on the SMC mitotic index, measured in vivo, builds on previous literature reporting the robust proliferation of SMCs in injured vessels. We measured high rates of mitosis in injured vessels and detectable mitoses even in uninjured arteries. The direct imaging and counting of cells in various mitotic stages in the artery in vivo gives a more accurate measure of cell proliferation compared with previous techniques based on the incorporation of radioactive or chemical agents during DNA synthesis, or the expression of cell cycle markers, which may be present for only short or variable times after the completion of cell division. ${ }^{18,19}$ Previous studies likely underestimated cell proliferation because they examined only a few nuclei in thin cross sections of the vessel, whereas our three-dimensional confocal technique detects all nuclei within the full thickness of the vessels and the $1 \mathrm{~cm}$ length of the carotid artery.

Cells with micronuclei were seen frequently in the neointima and immediately subjacent medial layers of ballooninjured arteries; interestingly, micronuclei have also been observed in the atherosclerotic plaques of patients. ${ }^{21}$ Micronuclei result when asymmetric cell division produces acentric chromosomes without spindle attachment, which are often excluded from the nucleus of daughter cells. The accumulation of such defects could lead to the arrest of cell division, which might slow plaque growth. However, it is equally possible that increases in apoptosis are triggered to eliminate these abnormal cells, which could lead to enhanced inflammatory responses and a greater incidence of plaque rupture. 

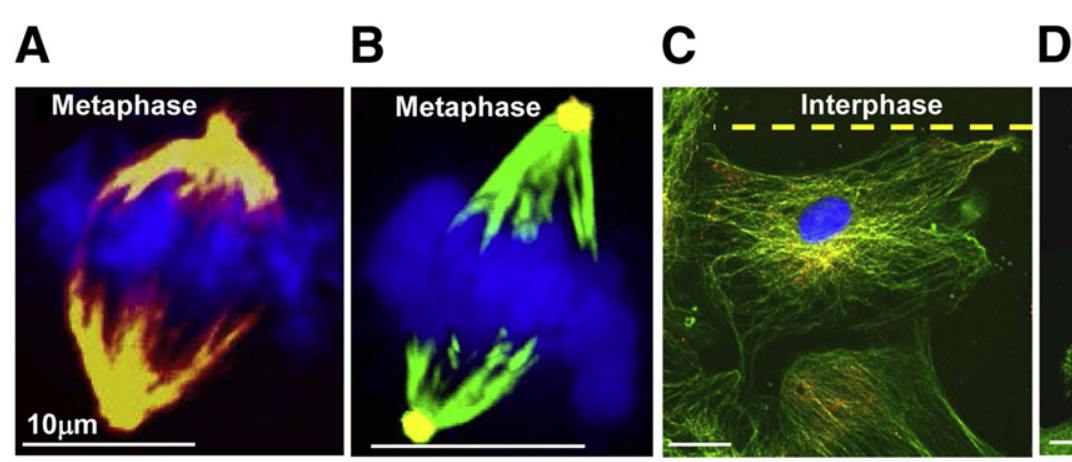

D

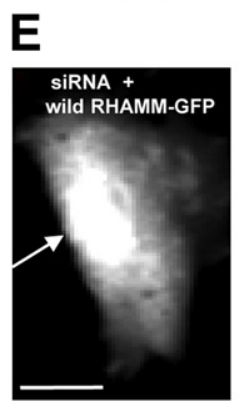

F

G
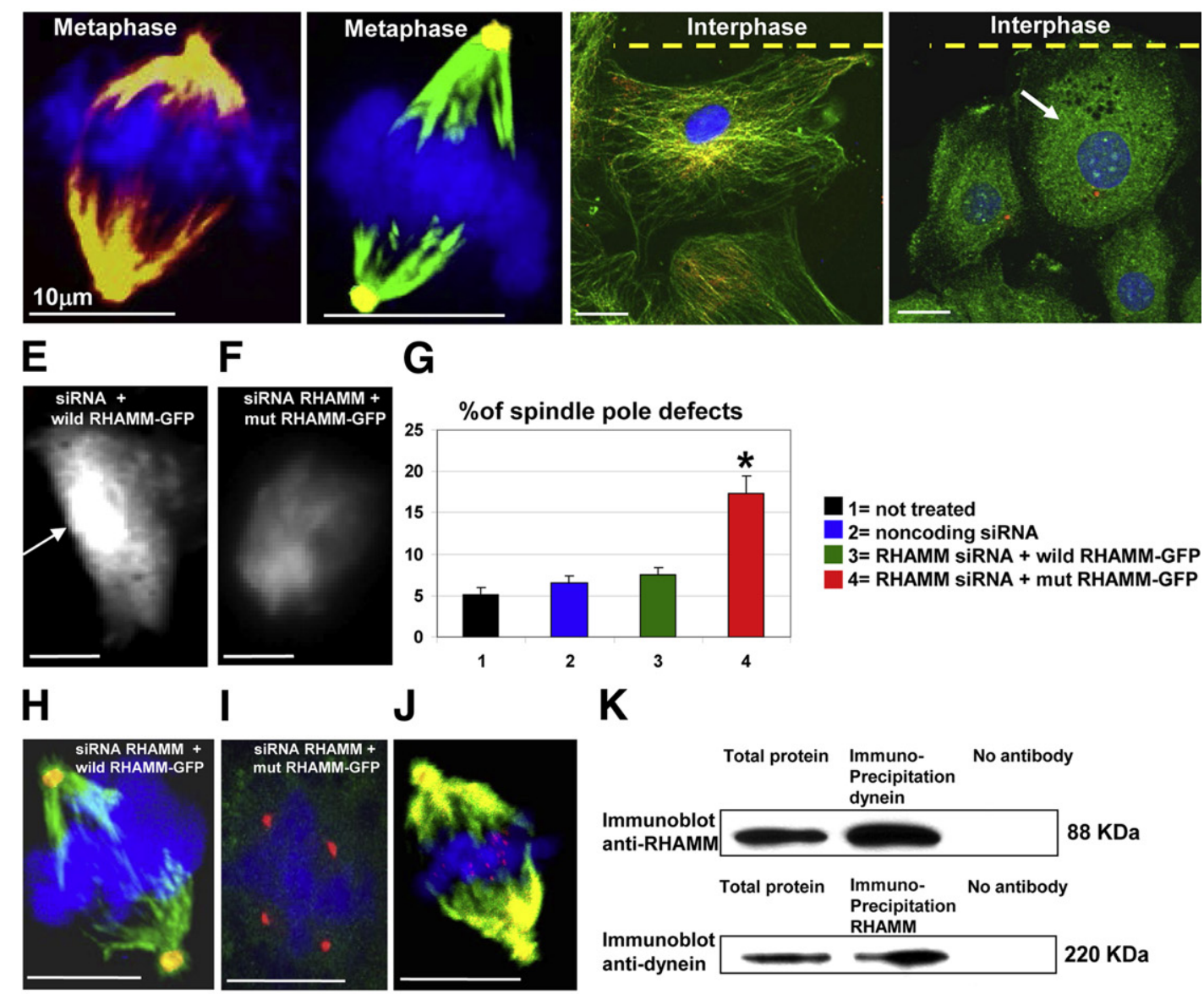

K
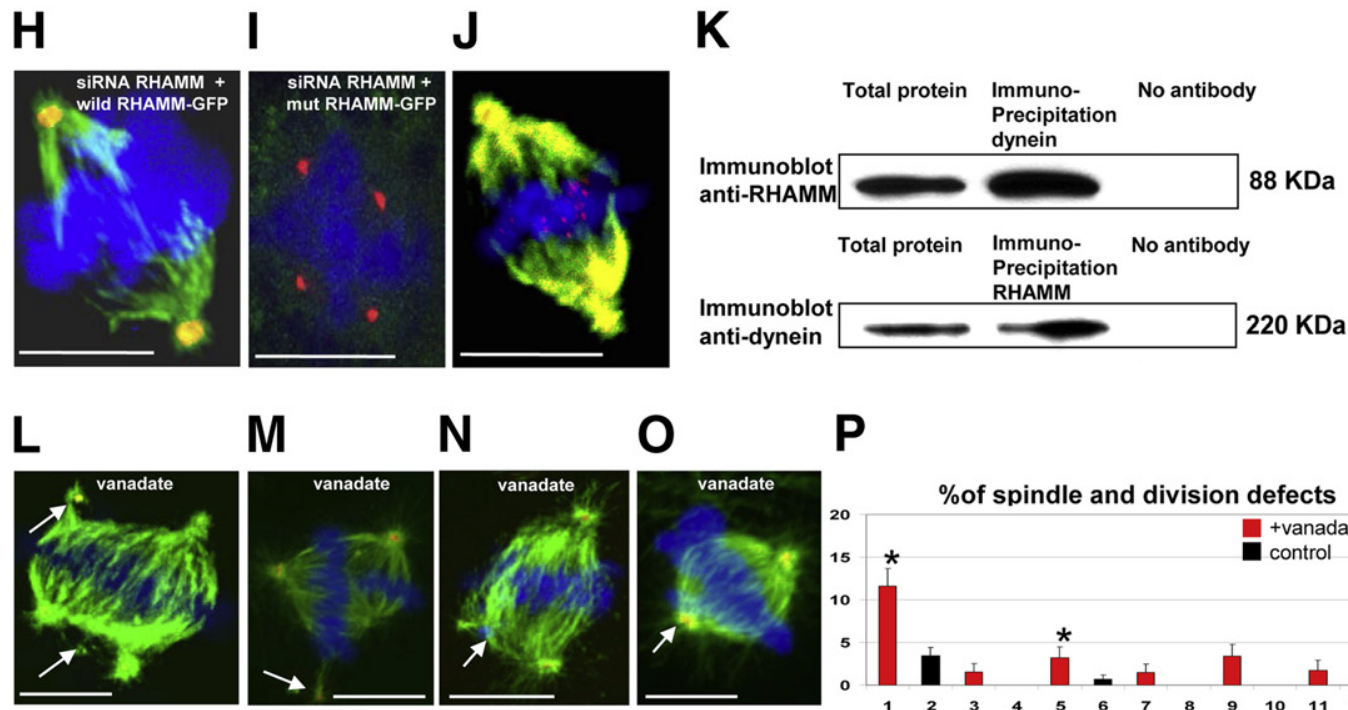

N

0

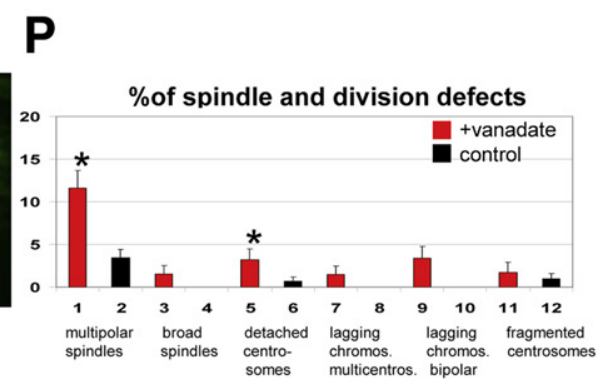

Q

$\mathbf{R}$

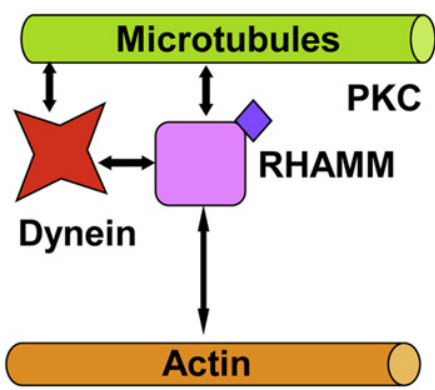

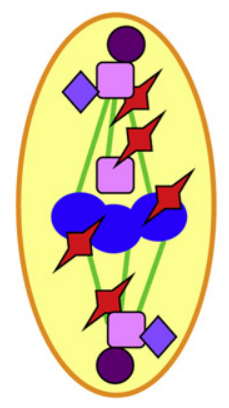

Karyokinesis

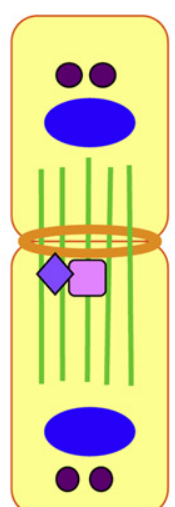

Cytokinesis
$2=$ noncoding siRNA

3= RHAMM SIRNA + wild RHAMM-GFP

4= RHAMM SIRNA + mut RHAMM-GFP
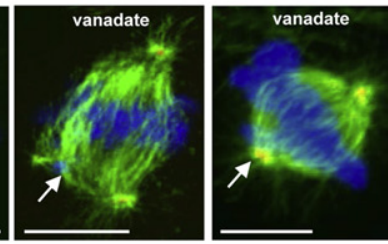

multipoler

multicentros, bipolar 
There were few binucleate cells in uninjured arteries, but a much higher frequency appeared in SMCs forming the neointima and in subjacent medial SMCs. Our data cannot discriminate whether this was due to cytokinesis failure because of defects in actin furrow assembly and constriction, or to defects in karyokinesis that triggered checkpoint signals blocking cytokinesis. However, we favor the second possibility because analysis of actin staining in neointimal SMCs in vitro did not reveal gross defects of the actin furrow and contractile ring. Rather, the spindle defects that we observed could account for defects in cytokinesis, because it is known that astral microtubules and the central spindle dictate the position of furrow assembly, ${ }^{22}$ and that the midbody is important for late cytokinesis and abscission. ${ }^{23}$ A previous study ${ }^{24}$ reported the presence of binucleate tetraploid smooth muscle cells in the human artery wall with a frequency that increased with aging; thus, the appearance of these cells can be the result of traumatic injury or a natural feature of vessel aging. Cancer cells are frequently binucleate and, in these populations, there are negative effects on cell viability and subsequent cell divisions; thus, most binucleate cells remain in interphase and never divide again. ${ }^{25}$ When binucleate cells enter mitosis, they contain three or more centrosomes, resulting in multipolar spindles and the failure of cytokinesis. ${ }^{26}$ They exhibit chromosome missegregation rates of up to 166-fold higher than the normal mitotic cell population. ${ }^{25}$

We previously showed that RHAMM was highly phosphorylated in neointimal compared with medial SMCs, and this was required for proper polarization of the centrosome and the migration of cultured neointimal SMCs. ${ }^{9}$ Moreover, we identified PKC $\alpha$ as one kinase responsible for RHAMM phosphorylation. In the current study, treating neointimal
SMCs in culture with PKC $\alpha$ inhibitor or using siRNA to knock down RHAMM, we demonstrate that RHAMM and $\mathrm{PKC} \alpha$ are also important in controlling the centrosome-based events of cell division. This is consistent with previous work showing that another isoform (namely, $\mathrm{PKC} \beta$ ) is localized at the centrosomes of various cell types and is involved in regulating the polarity of the microtubule cytoskeleton. ${ }^{27,28}$ Furthermore, PKC $\alpha$ is up-regulated during vascular SMC migration, ${ }^{29}$ and $\mathrm{PKC} \delta$ expression is elevated in human restenotic lesions and in the arterial wall in response to injury; it is a regulator of apoptosis in vascular SMCs. ${ }^{30}$ Moreover, our results are in accord with studies in fibroblasts showing that RHAMM knockdown led to multipolar spindles, aberrant chromosome segregation, and defects in cytokinesis. ${ }^{31}$ RHAMM has potent oncogenic potential: its ectopic expression results in the transformation and metastasis of fibroblasts, ${ }^{32}$ and high expression of RHAMM is a predictor of poor outcome in some tumors. ${ }^{33}$ Our data suggest that RHAMM and PKC $\alpha$ regulated division fidelity but did not affect cell entry into mitosis. This is not surprising in light of previous data that showed that $\mathrm{PKC} \delta$ is the PKC isoform mediating vascular smooth cell proliferation. ${ }^{34,35} \mathrm{In}$ addition, SMC proliferation (determined by cell counting or 5-bromo-2'-deoxyuridine incorporation) is independent of the hyaluronan-RHAMM signaling pathway. ${ }^{10}$

We found that mutation of the RHAMM centrosomaltargeting domain interfered with localization of this protein to the spindle poles, and with spindle assembly. This RHAMM motif is similar to the centrosomal-targeting motifs of other proteins involved in spindle organization (eg, TACC and Klp2). ${ }^{11}$ Moreover, RHAMM associates with spindle assembly proteins, such as TPX2, and is required for the activation of

\footnotetext{
Figure 4 The centrosomal-targeting domain of RHAMM and dynein are necessary for spindle organization and chromosome segregation. A-D: Confocal microscopy images showing immunostaining for RHAMM (green), DNA (blue), $\alpha$-tubulin (red in A and C), and $\gamma$-tubulin (red in B and D) in neointimal SMCs. Yellow indicates colocalization. The yellow dashed line in $\mathbf{C}$ and $\mathbf{D}$ indicates the wound. A : In metaphase, RHAMM is partially colocalized with spindle microtubules and is present at spindle poles (B). In interphase, RHAMM is concentrated in the perinuclear region (C and D). E and F: Live fluorescence microscope images of interphase neointimal SMCs treated with siRNA for RHAMM and transfected with RHAMM-GFP. E: After transfection with WT RHAMM-GFP, RHAMM is concentrated in the perinuclear region (arrow). F: After transfection with mutated RHAMM-GFP, there is less RHAMM in the cytoplasm and around the nucleus. $n=102$ neointimal SMCs transfected with wild RHAMM-GFP, $n=126$ neointimal SMCs transfected with mutated RHAMM-GFP, and $N=3$ repeats of a live imaging experiment. G: Graph showing the percentage of cells with a spindle pole defect in cells after treatment with siRNA for RHAMM and transfection with WT or mutated RHAMM-GFP. ${ }^{*} P \leq 0.05$. H and I: Confocal microscopy images of dividing neointimal SMCs stained for RHAMM (green), centrosome (red), and DNA (blue). H: Control SMCs in early anaphase transfected with WT RHAMM-GFP; RHAMM is localized at the spindle poles and in the area of the spindle. I: SMCs in anaphase transfected with mutated RHAMM-GFP; the cell exhibits a tetrapolar mitotic figure, and there is less RHAMM around centrosomes. J: Confocal microscopy image of a neointimal SMC in late prometaphase stained for RHAMM (green), dynein (red), and DNA (blue) shows the colocalization (yellow) of RHAMM and dynein in the spindle area. Dynein is also present in some of the kinetochore microtubules. K: Lysates prepared from neointimal SMCs were immunoprecipitated with an anti-dynein antibody or RHAMM antibody and then immunoblotted using an anti-RHAMM antibody or an anti-dynein antibody. A band at $88 \mathrm{kDa}$ was detected by anti-RHAMM antibody in the total lysate and the co-immunoprecipitation experiment. A band of $70 \mathrm{kDa}$ was detected by the anti-dynein antibody. The leftmost lane contained total cell lysate, and the rightmost lane was a negative control. The blots shown are representative of three independent experiments. L-0: Confocal microscopy images showing immunostaining for $\boldsymbol{\alpha}$-tubulin (green), DNA (blue), and $\gamma$-tubulin (red) in neointimal SMCs treated with the dynein inhibitor, vanadate. Yellow indicates colocalization. L: Multipolar spindle with centrosome clustering and broad spread microtubules, and detached centrosomes (arrows). M: Tripolar spindle with detached centrosome (arrow). N: Tripolar spindle with lagging chromosome (arrow). 0: Bipolar spindle with lagging chromosome (arrow). P: Graph showing the percentage of cells with spindle pole defects in cells after treatment with the dynein inhibitor, vanadate. 1 indicates multipolar spindles; 3, broad spindles; 5 , detached centrosomes; 7, lagging chromosomes in multicentrosomal spindles with a pseudobipolar architecture; 9, lagging chromosomes in bipolar spindles; 11, fragmented centrosomes. 2, 6, and 12 indicate control values taken from Figure 3. ${ }^{*} P \leq 0.05$ compared with control. Q-R: Model of the role of RHAMM, PKC $\alpha$, and dynein in mitosis. Q: Dynein and RHAMM are part of a complex. R: RHAMM and dynein regulate division spindle assembly and division fidelity (left). RHAMM also plays a role in cytokinesis (right). $n=$ 583 neointimal SMCs + siRNA for RHAMM + WT RHAMM-GFP, $n=629$ neointimal SMCs + siRNA for RHAMM + mutated RHAMM-GFP, $n=890$ neointimal SMCs + noncoding siRNA, $n=1002$ neointimal SMCs + siRNA for RHAMM, $n=172$ cells treated with dynein inhibitor vanadate, and $n=89$ cells stained for RHAMM and dynein. $N=6$ repeats of the fixed imaging experiment. Scale bar $=10 \mu \mathrm{m}$. Data indicate means \pm SD.
} 
Aurora A kinase, a protein with a role in chromosome division. ${ }^{36}$ The RHAMM centrosomal-targeting motif may also regulate interactions with dynein. Dynein plays a major role in spindle assembly by sliding oppositely oriented microtubules. ${ }^{37}$ Indeed, in human RHAMM, the centrosomal-targeting domain has $72 \%$ identity to the dynein interaction domain of the protein, XKLP2, and human RHAMM associates with centrosomes and interacts with dynein. ${ }^{11}$ An additional microtubule-binding domain has been shown at the $\mathrm{N}$ terminus of RHAMM, ${ }^{11}$ suggesting that RHAMM may cross-link centrosomal microtubules and facilitate the role of dynein in spindle organization.

In this study, we have shown important interactions between RHAMM and dynein in SMCs, illustrated schematically in Figure 4Q. The ability of RHAMM to interact with this microtubule motor suggests a mechanism by which RHAMM focuses and stabilizes microtubule bundles at the spindle poles in mitosis and, thus, coordinates normal centrosomal-based bipolar division spindle assembly and ensures division fidelity (Figure 4R). Indeed, our data show that dynein inhibition with vanadate in neointimal SMCs led to the formation of many defects, similar to those seen after RHAMM inhibition, including multipolar spindles, broader spindles, detached centrosomes, and lagging chromosomes in multipolar spindles with a pseudobipolar architecture. We propose a model (Figure 4R) in which RHAMM and its associated kinase, $\mathrm{PKC} \alpha$, together with dynein, act at centrosomes to regulate bipolar spindle assembly, focusing microtubules at spindle poles and maintaining the link between centrosomes and spindle microtubules at spindle poles. In contrast to the similarities between PKC/RHAMM and dynein inhibition, there were some defects that were seen only after dynein inhibition, such as an increased number of bipolar spindles with lagging chromosomes, or only after PKC and RHAMM inhibition (namely, centrosome fragmentation). These data suggest that dynein plays a role in chromosome movement along microtubules, important to their attachment to kinetochore fibers, congression at the metaphase plate, and chromosome movement in anaphase, whereas RHAMM and PKC play additional roles in maintaining the coalescence of centrosome components. RHAMM and its associated kinase, $\mathrm{PKC} \alpha$, are also important for cytokinesis, perhaps by stabilizing midbody microtubules and the contractile ring during cytokinesis (Figure 4R).

In conclusion, to our knowledge, this is the first detailed in vivo analysis of cell division defects during the SMC response to vascular injury. We show that increases in cell proliferation are accompanied by the loss of fidelity in cell division; this, in turn, is followed by increases in cell apoptosis. These processes could lead to the propagation of cells with damaged DNA and/or atherosclerotic plaque instability due to increased rates of SMC apoptosis. We have identified PKC $\alpha$, RHAMM, and dynein as important regulators of the fidelity of SMC division; they could serve as important biomarkers for cell transformation in atherosclerosis and have relevance for the development of therapeutic agents to reduce growth and increase stability of the atherosclerotic plaques.

\section{Acknowledgment}

We thank Dr. Milton Charlton for invaluable critical discussions, suggestions, and funding for the project.

\section{References}

1. Fuster JJ, Fernandez P, Gonzalez-Navarro H, Silvestre C, Naim Y, Nabah A, Andres V: Control of cell proliferation in atherosclerosis: insights from animal models and human studies. Cardiovasc Res 2010 , $86: 254-264$

2. Morganti M, Carpi A, Nicolini A, Gorini I, Glaviano B, Fini M, Giavaresi G, Mittermayer C, Giardino R: Atherosclerosis and cancer: common pathways on the vascular endothelium. Biomed Pharmacother 2002, 56:317-324

3. Li JJ, Gao RL: Should atherosclerosis be considered a cancer of the vascular wall? Med Hypotheses 2005, 64:694-698

4. Ross JS, Stagliano NE, Donovan MJ, Breitbart RE, Ginsburg GS: Atherosclerosis: a cancer of the blood vessels? Am J Clin Pathol 2001, 116:97-107

5. Benditt EP: Evidence for a monoclonal origin of human atherosclerotic plaques and some implications. Circulation 1974, 50:650-652

6. Gray K, Bennett M: Role of DNA damage in atherosclerosis: bystander or participant? Biochem Pharmacol 2011, 82:693-700

7. Carroll PE, Okuda M, Horn HF, Biddinger $\mathrm{P}$, Stambrook PJ, Gleich LL, Li Y-Q, Tarapore P, Fukasawa K: Centrosome hyperamplification in human cancer: chromosome instability induced by p53 mutation and/or Mdm2 overexpression. Oncogene 1999, 18: 1935-1944

8. Schvartzman JM, Sotillo R, Benezra R: Mitotic chromosomal instability and cancer: mouse modelling of the human disease. Nat Rev Cancer 2010, 10:102-115

9. Silverman-Gavrila R, Silverman-Gavrila L, Hou G, Zhang M, Charlton M, Bendeck MP: Rear polarization of the microtubuleorganizing center in neointimal smooth muscle cells depends on PKC $\alpha$, ARPC5, and RHAMM. Am J Pathol 2011, 178:895-910

10. Gouëffic Y, Guilluy C, Guérin P, Patra P, Pacaud P, Loirand G: Hyaluronan induces vascular smooth muscle cell migration through RHAMM-mediated $\mathrm{PI}_{3} \mathrm{~K}$-dependent Rac activation. Cardiovasc Res 2006, 72:339-348

11. Maxwell CA, Keats JJ, Crainie M, Sun X, Yen T, Shibuya E, Hendzel M, Chan G, Pilarski LM: RHAMM is a centrosomal protein that interacts with dynein and maintains spindle pole stability. Mol Biol Cel 2003, 14:2262-2276

12. Bendeck MP, Zempo N, Clowes AW, Galardy RE, Reidy MA: Smooth muscle cell migration and matrix metalloproteinase expression after arterial injury in the rat. Circ Res 1994, 75:539-545

13. Hou G, Mulholland D, Gronska MA, Bendeck MP: Type VIII collagen stimulates smooth muscle cell migration and matrix metalloproteinase synthesis after arterial injury. Am J Pathol 2000, 156:467-476

14. Callaini G, Riparbelli MG: Centriole and centrosome cycle in the early Drosophila embryo. J Cell Sci 1990, 97:539-543

15. Yang B, Zhang L, Turley EA: Identification of two hyaluronan-binding domains in the hyaluronan receptor RHAMM. J Biol Chem 1993, 268: 8617-8623

16. Shimizu T, Toyoshima YY, Edamatsu M, Vale RD: Comparison of the motile and enzymatic properties of two microtubule minus-enddirected motors, ncd and cytoplasmic dynein. Biochemistry 1995 34:1575-1582

17. Kunda P, Pelling A, Liu T, Baum B: Moesin controls cortical rigidity, cell rounding, and spindle morphogenesis during mitosis. Curr Biol 2008, 18:91-101 
18. Clowes AW, Reidy MA, Clowes MM: Kinetics of cellular proliferation after arterial injury, I: smooth muscle growth in the absence of endothelium. Lab Invest 1983, 49:327-333

19. Han DK, Haudenschild CC, Hong MK, Tinkle BT, Leon MB, Liau G: Evidence for apoptosis in human atherogenesis and in a rat vascular injury model. Am J Pathol 1995, 147:267-277

20. Bochaton-Piallat ML, Gabbiani F, Redard M, Desmouliere A, Gabbiani G: Apoptosis participates in cellularity regulation during rat aortic intimal thickening. Am J Pathol 1995, 146:1059-1064

21. Botto N, Rizza A, Colombo MG, Mazzone AM, Manfredi S, Masetti S, Clerico A, Biagini A, Andreassi MG: Evidence for DNA damage in patients with coronary artery diseases. Mutat Res 2001, 493:23-30

22. Glotzer M: Cleavage furrow positioning. J Cell Biol 2004, 164: 347-351

23. Steigemann P, Gerlich DW: Cytokinetic abscission: cellular dynamics at the midbody. Trends Cell Biol 2009, 19:606-616

24. Barrett TB, Sampson P, Owens GK, Schwartz SM, Benditt EP: Polyploid nuclei in human artery wall smooth muscle cells. Proc Natl Acad Sci U S A 1983, 80:882-885

25. Shi Q, King RW: Chromosome nondisjunction yields tetraploid rather than aneuploid cells in human cell lines. Nature 2005, 437: $1038-1042$

26. Wong C, Stearns T: Mammalian cells lack checkpoints for tetraploidy, aberrant centrosome number, and cytokinesis failure. BMC Cell Biol 2005, 6:6

27. Chen D, Purohit A, Halilovic E, Doxsey SJ, Newton AC: Centrosomal anchoring of protein kinase $\mathrm{C} \beta \mathrm{II}$ by pericentrin controls microtubule organization, spindle function, and cytokinesis. J Biol Chem 2003, 79: 4829-4839

28. Volkov Y, Long A, McGrath S, Eidhin DN, Kelleher D: Crucial importance of PKC-beta (I) in LFA-1-mediated locomotion of activated T cells. Nat Immunol 2001, 2:508-514

29. Herbert J-M, Clowes M, Lea HJ, Pascal M, Clowes AW: Protein kinase $\mathrm{C} \alpha$ expression is required for heparin inhibition of rat smooth muscle cell proliferation in vitro and in vivo. J Biol Chem 1996, 42: 25928-25935

30. Yamanouchi D, Kato K, Ryer EJ, Zhang F, Liu B: Protein kinase C delta mediates arterial injury responses through regulation of vascular smooth muscle cell apoptosis. Cardiovasc Res 2010, 85:434-443

31. Tolg C, Hamilton SR, Morningstar L, Zhang J, Zhang S, Esguerra KV, Telmer PG, Luyt LG, Harrison R, McCarthy JB, Turley EA: RHAMM promotes interphase microtubule instability and mitotic spindle integrity through MEK1/ERK1/2 activity. J Biol Chem 2010, 285: 26461-26474

32. Hall CL, Yang B, Yang X, Turley M, Samuel S, Lange LA, Wang C, Curpen GD, Savani RC, Greenberg AH, Turley EA: Overexpression of the hyaluronan receptor RHAMM is transforming and is also required for H-ras transformation. Cell 1995, 82:19-28

33. Wang C, Thor AD, Moore DH, Zhao Y, Kerschmann R, Stern R, Watson PH, Turley EA: The overexpression of RHAMM, a hyaluronanbinding protein that regulates ras signaling, correlates with overexpression of mitogen-activated protein kinase and is a significant parameter in breast cancer progression. Clin Cancer Res 1998, 4:567-576

34. Yamanouchi D, Kato K, Ryer EJ, Zhang F, Liu B: Protein kinase C delta mediates arterial injury responses through regulation of vascular smooth muscle cell apoptosis. Cardiovasc Res 2010, 85:434-443

35. Fukumoto S, Nishizawa Y, Hosoi M, Koyama H, Yamakawa K, Ohno S, Morii $\mathrm{H}$ : Protein kinase $\mathrm{C}$ delta inhibits the proliferation of vascular smooth muscle cells by suppressing G1 cyclin expression. J Biol Chem 1997, 272:13816-13822

36. Hatano $H$, Shigeishi $H$, Kudo $Y$, Higashikawa K, Tobiume K, Takata T, Kamata N: RHAMM/ERK interaction induces proliferative activities of cementifying fibroma cells through a mechanism based on the CD44-EGFR. Lab Investig 2011, 91:379-391

37. Gatlin JC, Matov A, Groen AC, Needleman DJ, Maresca TJ, Danuser G, Mitchison TJ, Salmon ED: Spindle fusion requires dyneinmediated sliding of oppositely oriented microtubules. Curr Biol 2009, 19:287-296 